Alma Mater Studiorum - Università di Bologna DEPARTMENT OF ECONOMICS

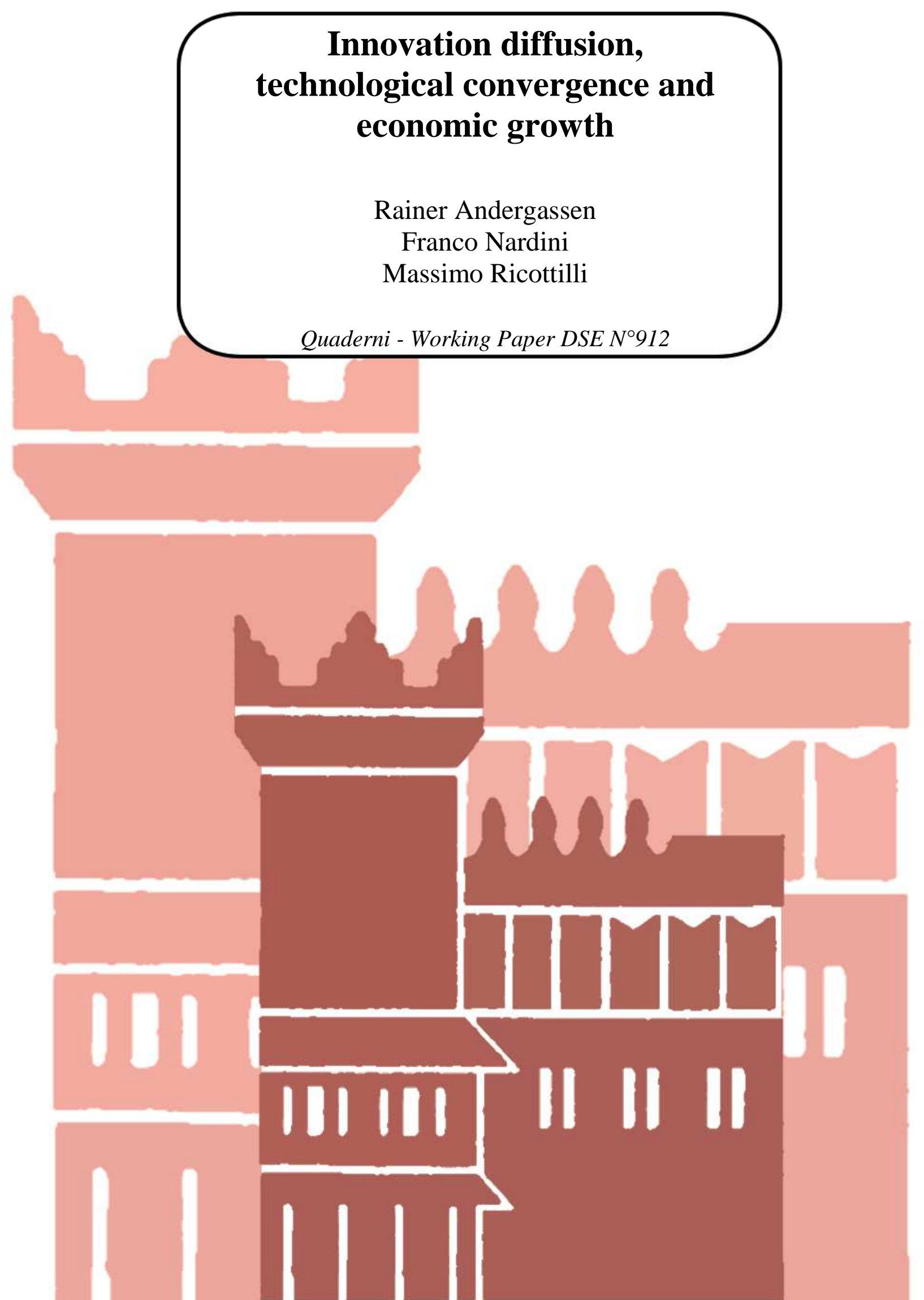




\title{
Innovation diffusion, technological convergence and economic
}

$$
\text { growth* }
$$

\author{
Rainer Andergassen, Franco Nardini, Massimo Ricottilli \\ University of Bologna
}

November 20, 2013

\begin{abstract}
The paper investigates the mechanics through which novel technological principles are developed and diffused throughout an economy consisting of a technologically heterogeneous ensemble of firms. In the model entrepreneurs invest in the discovery and in the diffusion of a technological principle and their profit flow depends on how many firms adopt the innovation and on how long it takes other entrepreneurs to improve it. We show that technological convergence emerges from the competition among entrepreneurs for the profit flow and characterize the economy's growth rate.

JEL: O31; O33; O410; E190
\end{abstract}

Keywords: innovation; innovation diffusion; technological convergence; general purpose technologies; economic growth

*1. This paper has been written with the support of a 'PRIN 2009' grant provided by MIUR (Italian Ministry of Education, University and Research) as part of the research project "Local interactions and global dynamics in economics and finance: models and tools" 


\section{Introduction}

Technological diffusion is a crucial factor in fostering productivity growth. It is worth noting, however, that this process is not merely the replication and imitation of known and well established techniques, although this may be a substantial part of the whole, but is characterized by a sequence of innovations through which the technology is spread across different firms belonging to different production sectors. Whilst the most radical form taken by this process is the diffusion of a general purpose technology (see for example the discussion in David, 1991; Helpman and Trajtenberg, 1998; Helpman, 1998; Rosenberg, 1976, 1982; Rosenberg and Trajtenberg, 2004), it also involves the piecemeal adaptation of new artifacts to different usages and productive purposes. In any case, it clearly hinges on knowledge and information transmission. As early as 1958, March and Simon (1958), in a seminal contribution concerning the functioning of organizations, held that much innovation results from borrowed knowledge, that is from knowledge firstly developed in other firms or in other industries. More recently, this important theme has been further investigated by the work of Cohen and Levinthal $(1989,1990)$. Their case is based on the well tested argument that new knowledge is strongly dependent on previously accumulated knowledge. Furthermore, these authors argue that firms that carry out and invest in R\&D are capable of adapting knowledge originating in other firms. This is clearly a process that accounts for much diffusion and ultimately for technological convergence. ${ }^{1}$

The purpose of this paper is to characterize the growth rate of an economy featuring a high degree of heterogeneity both in terms of output variety and of the technologies that are accordingly employed and to investigate the mechanics through which a single technological principle is introduced throughout the whole economy. We show that entrepreneurs' investments, aimed at discovering a technological principle and diffusing it throughout the economy, together with the competition among entrepreneurs for the profit flow leads to the emergence of technological convergence.

Proximity in our model is defined in terms of technological distance. Although much literature has dealt with geographical networks and clustering, consider for example the rich spate of contributions on industrial districts, we take the view that in light of the new means provided by information technologies what matters most for innovation diffusion is technological rather than geographical proximity (see also Andergassen, Nardini and Ricottilli, 2006). At least for the purpose of investigating innovation diffusion, situations where the introduction of the new technological principle needs only minor innovations, that is, a mere adaptation, are distinguished from situations where major innovations are

\footnotetext{
${ }^{1}$ See Fai and Von Tunzelmann (2001) for empirical evidence on technological convergence.
} 
required. Historical evidence has in fact indicated that the effective diffusion of a new technological principle has often required enabling and essential complementary innovations (Goldfarb 2005). We envisage a clustering principle responding to a criterion of technological proximity shaped by the inherent problems they face, the corresponding skills and expertise they possess. The economy that results from this view of firm heterogeneity is an ensemble of clusters that differ in terms of their technological profile, each collecting firms that produce different things but that are technologically alike. Within each cluster, firms are still heterogeneous in terms of their performance and the goods they produce but exhibit a high degree of technological likeness. It is important to stress that the proximity of technological clusters is not defined at the beginning of the process but depends on the evolution of the technology.

We distinguish two types of investments. Investment aimed at the discovery of the technological principle and investment aimed at diffusion throughout the economy. While the first in quite standard, the second one is the upshot of the assumption that firms employ heterogeneous technologies and hence diffusion implies that the original innovation must be adapted to the specific needs of a new user, even in the case of technological proximity. We assume that investments in within-cluster diffusion gives rise to a learning-by-doing principle, that may lead to ideas and opportunities to introduce the technological principle into firms belonging to other technological clusters ${ }^{2}$. In other words, it is conjectured that technological opportunities to successfully find applications in distant clusters emerge as a consequence of innovative investment that leads, on the one hand, to score success within clusters and, on the other, as a consequence of learning, generates technological opportunities to cross over to other clusters. The learning-to adapt process lays the ground for a success breeds success feedback but it is a necessary condition not a sufficient one. Leaping across technological barriers is an effort of a very challenging nature and quite distinct from that required to within-cluster diffusion.

Our paper is related to Silverberg and Verspagen (2005) where technological trajectories and innovation dynamics are studied in a model of percolation in a complex technology space and Arenas et al. (2002) where the dynamics of innovation are analyzed with a model of local interaction. Andergassen, Nardini and Ricottilli $(2006,2009)$ investigate in a model of innovation diffusion and economic growth with local interaction among heterogeneous firms the conditions for the emergence of technological convergence. While in these papers the set of technological neighbors is exogenously defined, in this

\footnotetext{
${ }^{2}$ Investment in the diffusion within a technology cluster may also lead to a reduction of costs as a consequence of a learning-by-doing effect. Hence, the greater the number of within-cluster adoptions the lower the costs and therefore the greater the likelihood of introducing the technological principle in other clusters.
} 
one technological proximity is endogenously determined, depending on the characteristics and the unfolding of the technology development process. Moreover, we show that technological convergence is the result of investment in technology diffusion and the competition among entrepreneurs for the profit flow.

The remaining part of the paper is organized as follows. In Section 2 we discuss the innovation process. In particular, we describe technological clusters, the process of within- and between-cluster diffusion, the time and space dimension of the innovator's profit flow and determine the innovator's incentive to invest in the discovery and in the diffusion of the technological principle. In Section 3 we characterize the resulting emergent properties of the diffusion process and the features of economic growth. In Section 4 we generalize the model by considering the diffusion of heterogeneous technological principles and the competition among an endogenously determined number of entrepreneurs that invest in $R \& D$. Section 5 draws some conclusions.

\section{The innovation process}

The economy we wish to deal with is composed of $J$ final goods each of which is produced by a firm with a specific technique and monopoly power. We consider an $R \& D$ race of vertical non-drastic innovations where the successful innovator replaces the incumbent. In particular, we distinguish two innovation processes: the discovery of a novel technological principle and its diffusion throughout the economy. Both are ultimately chance events the key of which is the investment that the innovating entrepreneur is willing to devote to these tasks and they are accordingly modeled by a probability structure that depends on investment. We start by considering the efforts of just one innovator discussing later the competition that arises thanks to a sequence of further competing productivity increasing innovations. As a matter of reference and for simplicity's sake, the economy we discuss can be viewed as one in which there is a representative consumer with the following utility function:

$$
U\left(x_{1}, \ldots, x_{J}\right)=\sum_{j=1}^{J} \ln \left(x_{j}\right)
$$

where $x_{j}$ denotes the quantity of good $h$ bought. Normalizing the consumer's income to one, from the optimal consumption choice $\zeta_{j} x_{j}=1$, where $\zeta_{j}$ is the price of product $j$.

We first start describing the diffusion process and afterward we discuss the discovery of the novel 
technological principle.

\subsection{Technological clusters and diffusion}

It is expedient to group the $J$ final goods according to their technological proximity in order to form clusters of technological neighbors. These clusters are to be understood as ensembles of final goods that employ similar techniques. Within each, the goods that compose them are not homogeneous, they are all produced by specific processes but the difference in terms of the technological principles employed across clusters is far greater than within each. As stated in the introduction, the diffusion of a technological principle is a matter of innovative adaptation within a cluster and a matter of innovative application across clusters. The distance separating the latter is accordingly not apriori$l y$ known but depends on the improvement and development of the technological principle that is capable of achieving a cross-over linking clusters that may not have any beforehand likeness. There may, therefore, be innovations that prove easily introducible whilst others do not; some innovations that diffuse speedily in some clusters but do not in others or find barriers forbidding any diffusion. A technologically connected network is the result of the process, not its prerequisite. For the sake of simplicity we assume that each cluster is composed of $N$ goods, the whole economy to be a set of $\frac{J}{N}$ clusters and consider the limit $J \rightarrow \infty$ with a finite number of goods $N$ in each.

\subsection{Within-cluster diffusion}

Innovations lead to non-drastic productivity increases of size $\gamma>1$. Let $\kappa$ be the marginal production cost of an incumbent firm, then the marginal production costs of an outsider firm adopting the innovation are $\gamma^{-1} \kappa$. Because of competition between the insider and the outsider, the price charged by firm $h$ adopting the technology is $\zeta_{h}=\kappa$ and firm profits are

$$
\pi_{h}=\theta^{\circ}-c_{h}
$$

where $\theta^{\circ} \equiv 1-\gamma^{-1}$ and where $c_{h}$ are fixed costs (per unit time) of the new technology. Successful implementation of the original technological principle depends thus on the size of the fixed costs. We designate by $I$ the investment expenditure aimed at reducing them. We assume that for each final good producer $h$, fixed $\operatorname{costs} c_{h}, h=1,2, \ldots, J$, are i.i.d. random variables that take the value $\theta^{\prime}<\theta^{\circ}$ with probability $p(I)$ and $\theta^{\prime \prime}>\theta^{\circ}$ with probability $1-p(I)$, where we assume that $p(I)$ is an increasing 
and concave function of $I$, with $p^{\prime}(I)>0$ for each $I<\infty$. Adoptions occur as long as $\theta^{\circ} \geq c_{h}$, it then follows that the mean number of adoptions is $\hat{n}(I)=N p(I)$, where $\hat{n}(I)$ is also increasing and concave in $I$. We define $\theta=\theta^{\circ}-\theta^{\prime}$.

\subsection{Between-cluster diffusion: the role of investment and diffusivity}

Every economic system lends itself in varying degrees to the diffusion of new technological principles.

It is its structure, the degree of development and its technological capabilities that set the framework enabling an innovation to diffuse. We term this feature of the diffusion process diffusivity and represent it by a single parameter $d .^{3}$

Reaching out to other clusters after the root one has been invaded requires the opening of technological opportunities, provided that the original innovation takes off from the drawing board. These paths away from a root cluster pry open with a probability that depends on the number of adoptions in the root cluster, a case of learning by adopting. Learning-by-adopting is of the utmost importance since by being conducive to the establishment of user-producer linkages, it is the instrument to gain knowledge and expertise that may point the way to other clusters; at every new adoption the technological principle widens its scope and the capability that is associated with it rises opening up new technological opportunities. For simplicity's sake we assume that this effect is captured by the mean number of adoptions. ${ }^{4}$.

Assumption 1 The mean number $\mu(I, d)$ is a concave increasing function with respect to $I$ and $d$, with $\mu(0, d)<1$ and $\lim _{I \rightarrow \infty} \mu(I, d)>1$.

The innovating entrepreneur will consider investment as a tool to maximize profits. I plays a double role generating, on the one hand, adoptions of innovations within a cluster and, on the other, through the effect of learning by adopting, increasing the mean number of new clusters that the innovator expects to reach. In this sense, it is a case of success breeds success.

\footnotetext{
${ }^{3} \mathrm{~A}$ more realistic hypothesis is to assume that diffusivity varies from cluster to cluster and is not a priori-ly known to the innovator. In this case, it can be assumed that diffusivities are i.i.d. random variables of which the innovator may only conjecture the cumulative distribution function $G(d, a)$, smooth and decreasing in $a$, where $a$ is a measure of diffusivity intensity: a property of the technological state of the economy which can be taken as known by the innovator. In this case $\mu(I, d)=\int_{0}^{+\infty} E(k \mid d=x) d_{x} G(x, a)$ where $E(k \mid d=x)=\int k d_{k} F(k, I, x)$ and under appropriate assumptions about concavity, qualitative results found below still hold.

${ }^{4}$ Assumption 1 is the natural consequence of the fact that the expected number of adopters within the cluster is increasing with respect to investment and that "success breeds success". In the Appendix we restate Assumption 1 providing conditions such that $\mu(I, d)$ be a concave increasing function with respect to $I$ and $d$.
} 


\subsection{Investment and the diffusion of innovations}

The innovator's profit flow has two dimensions: one in time and one in space. The profit flow stemming from a single adoption continues until a new technological principle is successfully introduced by an entrant which thereby ousts the current incumbent. This event occurs with probability $\lambda$ (over a unit time period) and clearly depends on the joint probabilities that a new seeding technological principle appears and that it diffuses originating adoptions and applications both within and across clusters. The size of the innovator's profit flow depends also on the number of within and between cluster adaptations. $\lambda$ will later be fully and consistently endogenously characterized.

Let $r$ be the long-term risk-neutral interest rate that defines the macroeconomic environment and which can be interpreted as the financial cost to be borne when investment is undertaken. ${ }^{5}$

Expected profits from the introduction of an innovation within a cluster are then $\pi(I, \lambda)=\frac{N p(I)}{r+\lambda} \theta$, which is a strictly increasing and concave function of $I$.

The diffusion process between clusters can be viewed as a branching process. Profits that the innovator expects to gain once step $s$ of the diffusion process has been reached depend both on the within-cluster expected profits and on the expected profits that can be made by reaching out to clusters one step further, the $s+1$-th: the former are given by $\pi(I, \lambda)$ and let the latter be designated by $V_{s+1}$. Note that expected profits in a given cluster do not depend on $s$. The innovator's problem is then to establish the amount of investment that determines the distribution of fixed production costs, and hence the number of adoptions within a cluster, and the expected number of new clusters that are likely to be reached.

How far an innovation can penetrate into complex, heterogeneous economies does not solely depend on their structural characteristics. Highly industry-specific technologies are less likely to pass across clusters and, therefore, the time it takes for an adoption transfer to be achieved can be assumed to be lengthier than in the case of technologies that are intrinsically easier to adapt. In this sense, an innovator must take into account the implied diffusion time span and discount the probability of successfully bridging any two given clusters. Let $\tau$ be this expected time, we denote then with $\rho=e^{-r \tau}$ the related discount factor. The slower is the technology likely to diffuse on account of its intrinsic characteristics, the larger $\tau$ and hence the lower the discount factor $\rho$.

\footnotetext{
${ }^{5}$ Assuming that consumers face an intertemporal consumption choice problem with utility function $\int_{0}^{\infty} e^{\sigma t} \sum_{j=1}^{J} \ln \left(x_{j, t}\right) d t$, where $\sigma$ is the intertemporal discount factor, then the balanced long term growth path requires that $r=\sigma$.
} 
Denoting by $V_{s}$ the value of the innovation at the $s$-th step, the innovator's problem can be framed as:

$$
V_{s}=\max _{I \geq 0}\left\{\pi(I, \lambda)+\rho \mu(I, d) V_{s+1}-I\right\}
$$

which recursively defines the value of an innovation. The first term in curly brackets represents expected profits within a given technology cluster, while the second term describes expected discounted profits from diffusing the innovation to other technology clusters. Both terms depend on investment since the latter reduces the probability of high fixed costs within a cluster thereby increasing the probability of within-cluster adoptions and, because of learning-by-doing, it opens up new avenues for adaptions into technologically distant clusters.

To insure that a unique solution to this problem exists, we must assume the following.

Assumption 2 The function $\pi(I, \lambda)+\rho \mu(I, d) V_{s+1}$ is concave in $I$.

Assumption 2 is far from restrictive since it is satisfied provided that $\pi(I, \lambda)$ is sufficiently concave.

Since at every step the outlook for further diffusion is the same as at the preceding ones, the expected values of $V_{s+1}$ and $V_{s}$ are equal, and (3) becomes

$$
V=\max _{I \geq 0}\{\pi(I, \lambda)+\rho \mu(I, d) V-I\}
$$

FOC for the maximum in (4) is simply:

$$
\frac{\partial \pi(I, \lambda)}{\partial I}+\rho \frac{\partial \mu(I, d)}{\partial I} V-1=0
$$

from which $I=I(V, \rho, d, \lambda)$ is obtained. Consequently we can write (4) as

$$
V=\psi(V, \rho, d, \lambda)
$$

where

$$
\psi(V, \rho, d, \lambda)=\pi(I(V, \rho, d, \lambda), \lambda)+\rho \mu(I(V, \rho, d, \lambda), d) V-I(V, \rho, d, \lambda)
$$

A sufficient condition for the existence of a unique solution for (6) is that the function $\psi$ in (7) is a 
contraction with respect to $V$. Using the envelope theorem

$$
\frac{\partial \psi(V, \rho, d, \lambda)}{\partial V}=\rho \mu(I(V, \rho, d, \lambda))
$$

from which the following sufficient condition for the existence of a solution to (4) is obtained:

$$
\rho \mu(I(V, \rho, d, \lambda), d)<1
$$

In the following we assume that parameters $\rho$ and $d$ are such that this condition is always satisfied and that $\theta$ is sufficiently large such that $V$ is always positive.

Let $V^{*}=V(\rho, d, \lambda)$ be the solution of (6) and $I^{*}(\rho, d, \lambda)=I(V(\rho, d, \lambda), \rho, d, \lambda)$. The following Lemma holds.

Lemma 1 (i) $\frac{\partial V^{*}}{\partial \rho}>0, \frac{\partial V^{*}}{\partial d}>0, \frac{\partial V^{*}}{\partial \lambda}<0$ and (i) $\frac{\partial I^{*}}{\partial \rho}>0, \frac{\partial I^{*}}{\partial d}>0, \frac{\partial I^{*}}{\partial \lambda}<0$

Proof of Lemma 1. Consider first part (i). Using the envelope theorem it is easy to see that $\frac{\partial \psi}{\partial \rho}>0$, $\frac{\partial \psi}{\partial d}>0$. Applying the implicit function theorem to $(6)$ one obtains $\frac{\partial V^{*}}{\partial \rho}=\frac{\frac{\partial \psi}{\partial \rho}}{1-\frac{\partial \psi}{\partial V}}>0$, $\frac{\partial V^{*}}{\partial d}=\frac{\frac{\partial \psi}{\partial d}}{1-\frac{\partial \psi}{\partial V}}>0$ and $\frac{\partial V^{*}}{\partial \lambda}=\frac{\frac{\partial \psi}{\partial \lambda}}{1-\frac{\partial \psi}{\partial V}}<0$. Let us rewrite FOC $(5)$ as $\Theta=0$, where $\Theta=\frac{\partial \pi(I, \lambda)}{\partial I}+\rho \frac{\partial \mu(I, d)}{\partial I} V-1$. Because of the second order condition $\Theta_{I}<0$ and applying the implicit function theorem we obtain $\frac{\partial I^{*}}{\partial \rho}=-\frac{\Theta_{\rho}}{\Theta_{I}}>0, \frac{\partial I^{*}}{\partial d}-\frac{\Theta_{d}}{\Theta_{I}}>0, \frac{\partial I^{*}}{\partial \lambda}=-\frac{\Theta_{\lambda}}{\Theta_{I}}<0$.

Whilst the first two partial derivatives are quite straightforward to interpret, the third is a reminder that the innovation value decreases when the probability of new innovations emerging as a result of entrants' success increases. The partial derivatives with respect to investment help to shed light on the role of the diffusivity parameters, $d$ and $\rho$. As it is to be expected, the higher is $d$, that is, the proner is the economy to diffusion on account of its inner technological characteristics, the larger is investment. This is, of course, due to the positive impact on profits that can be expected by exploiting the innovative principle in a favorable technological context. The lower is $\tau$, that is, the higher is $\rho$, the easier it is for a technology to bridge heterogeneous technology clusters, due to the positive impact on the value of innovation, the larger the investment. The larger is the probability of success of a new entrant $(\lambda)$, the lower is the value of an innovation since entrepreneurs expect to profit from it for a shorter time span, and thus the lower the investment in within and between cluster adaptations. 


\subsection{The discovery of the technological principle}

If any diffusion is to occur in this economy a seed innovation must appear. As in the case discussed above, those who partake in the race to innovate stand a chance of success that must be grounded on the amount of initial investment that they are willing to undertake for this very purpose. Within and across cluster diffusion depends upon this initial successful event as well as on the specific investment intended to support it. It is accordingly envisaged that such an initial innovation occurs according to a Poisson arrival rate that depends on the intensity of investment specifically intended to research and initially develop.

Let such a function be defined by $\phi(H)$, with the usual properties $\phi^{\prime}>0$ and $\phi^{\prime \prime}<0, H$ being the investment magnitude. Furthermore, making an innovation market ready and potentially applicable to a production process requires a further sunk cost, for instance the making of a prototype, the definition of an operative book of blueprints, etc. Let this cost be designated by $F$. This is the crucial first step in the chain of events likely to lead to productivity growth and to the eventual establishment of a true and generalized technological paradigm. It is, however, important to note that this investment expenditure and the activity it supports are to be distinguished from those that are conducive to learning-to-adapt and thus to within and between cluster diffusion. The incentive to engage in this groundwork activity is precisely the value of the innovation and it is accordingly assumed that the magnitude of investment $H$ results from the following maximum problem:

$$
\Pi=\max _{H} \phi(H) V(\rho, d, \lambda)-H-F
$$

Since $V(\rho, d, \lambda)$ is the solution of $(6)$, the first order condition yields $H^{*}(\rho, d, \lambda)$ and consequently $\phi(\rho, d, \lambda)$ is the probability that a new technological principle is discovered. Using Lemma 1, the concavity assumption of $\phi$ and the implicit function theorem it can be shown that $H^{*}$ is increasing in $\rho$ and $d$ and decreasing in $\lambda$. We assume that $F$ is sufficiently low such that $\Pi>0$.

\section{Innovation diffusion and economic growth}

Whilst $\rho, d$, are parameters that pertain, the former, specifically to the type of the new technology, the latter to the economy's structure, $\lambda$ is endogenously determined since it is the arrival rate of an innovation applied to a single final good. This event is generated by an entrant that if successful can 
terminate the incumbent's flow of profits. As such, it is an arrival rate that is jointly determined by two components: the probability of a seed innovation $\phi(H)$ and the probability that an innovation be adopted in the production of a single good. The latter can be derived from the expected number of adoptions, that is by the expected number of firms that adopt over the total number of firms, $J$. This magnitude can, in turn, be computed by considering the average number of adopters in each cluster multiplied by the expected number of clusters that the innovation is likely to reach. As shown above, the former is $p(\rho, d, \lambda) N$ while the latter can be obtained recursively. The average number of clusters reached at stage $s$ given that $z_{s-1}$ have been reached at stage $s-1$ is:

$$
E\left(z_{s}\right)=\mu(\rho, d, \lambda) z_{s-1}
$$

and thus $E\left(z_{s}\right)=\mu(\rho, d, \lambda)^{s}$. Summing over all the possible stages $G$, dividing by $J$ and taking into account that the average number of within-cluster adoptions is $p(\rho, d, \lambda) N$, the probability that the innovation reaches a particular final good is $p(\rho, d, \lambda) \frac{N}{J} \sum_{s=1}^{G} \mu(\rho, d, \lambda)^{s} \cdot \frac{N}{J} \sum_{s=1}^{G} \mu(\rho, d, \lambda)^{s}$ is also the expected proportion of all clusters that are likely to be reached.

Since the economy considered is very large, in the limit $J \rightarrow \infty$, we can consistently take the limit $\lim _{J \rightarrow \infty} \frac{N}{J} \sum_{s=1}^{G} \mu(\rho, d, \lambda)^{s}$. It is immediate to see that it depends on $\mu(\rho, d, \lambda) \gtrless 1$. If it is smaller than 1 , then branching is very short, the number of clusters reached is scant and the share of firms that are likely to adopt vanishing small for $J$ that tends to infinity: the limit is accordingly zero; if on the other hand, it is greater than 1 , then there exists a positive and finite limit: $\Delta(\rho, d, \lambda)$, i.e.

$$
\lim _{J \rightarrow \infty} \frac{N}{J} \sum_{s=1}^{G} \mu(\rho, d, \lambda)^{s}=\left\{\begin{array}{cccc}
\Delta(\rho, d, \lambda)>0 & \text { for } & \mu(\rho, d, \lambda)>1 \\
0 & \text { for } & \mu(\rho, d, \lambda)<1
\end{array}\right.
$$

Note that $\lim _{J \rightarrow \infty} \frac{N}{J} \sum_{s=1}^{G} \mu(\rho, d, \lambda)^{s}$, which is the proportion of clusters likely to be reached $b y$ the technological principle, is also the survival probability of the branching process. If $\mu(\rho, d, \lambda)<1$ then such a proportion is negligibly small and the branching process dies out with certainty; if $\mu(\rho, d, \lambda)>1$, then $\Delta$ is positive; equivalently, $\Delta$ is the probability that all clusters be reached.

On the strength of this result, a solution, $\lambda^{*}$ can consistently be obtained by solving the fixed point problem

$$
\lambda=\left\{\begin{array}{ccc}
\phi(\rho, d, \lambda) \Delta(\rho, d, \lambda) p(\rho, d, \lambda) & \text { for } & \mu(\rho, d, \lambda)>1 \\
0 & \text { for } & \mu(\rho, d, \lambda)<1
\end{array}\right.
$$


Note that on account of the concavity of functions $\mu$ and $p$ and since $\frac{\partial V}{\partial \lambda}<0$ and $\frac{\partial I}{\partial \lambda}<0, \frac{\partial \phi}{\partial \lambda}<0$, $\frac{\partial p}{\partial \lambda}<0$ and $\frac{\partial \Delta}{\partial \lambda}<0$; hence, the right hand side of the equation for $\mu(\rho, d, \lambda)>1$ is strictly decreasing in $\lambda$ leading to the conclusion that a positive $\lambda^{*}$ exists. In consequence of this solution the economy is fully characterized by only two exogenous parameters, one structural, $d$, and one technology-specific, $\rho$.

Proposition 1 For sufficiently low $r$ the unique solution to the fixed point problem (10) is $\lambda^{*}>0$ which solves $\lambda^{*}=\phi\left(\rho, d, \lambda^{*}\right) \Delta\left(\rho, d, \lambda^{*}\right) p\left(\rho, d, \lambda^{*}\right)$, where $\mu\left(\rho, d, \lambda^{*}\right)>1$ and $\Delta\left(\rho, d, \lambda^{*}\right)>0$. The corresponding economy's growth rate is

$$
g=\frac{\gamma-1}{\gamma} \phi(\rho, d) p(\rho, d) \Delta(\rho, d)
$$

which is increasing in $d$ and $\rho$ since $\phi\left(\rho, d, \lambda^{*}\right) \Delta\left(\rho, d, \lambda^{*}\right) p\left(\rho, d, \lambda^{*}\right)$ is increasing in $\rho$ and $d$.

Proof of Proposition 1. Consider the case where $r \rightarrow 0$ and suppose that $\lambda=0$, implying full monopoly power enjoyed by the innovator. As a consequence $\frac{\partial}{\partial I} \pi(I, \lambda)=\frac{N \theta}{r+\lambda} p^{\prime}(I) \rightarrow \infty$, with the consequence that the optimal investment becomes infinitely large $I^{*} \rightarrow \infty$ and thus $\mu\left(I^{*}, d\right)>1$, proving that $\lambda=0$ is not an equilibrium solution.

To clarify this point, consider the following extreme case. For $r \rightarrow 0$, implying an inexhaustible source of financial support, and suppose that $\lambda=0$, implying full monopoly power enjoyed by the innovator, marginal gains from investments diverge to infinity. As a consequence, the amount invested in the diffusion of the technological principle by the innovator will be infinitely large and thus the average number of clusters reached cannot be lower than 1 , implying that $\lambda=0$ cannot be an equilibrium. Therefore, for sufficiently low $r$ the unique equilibrium outcome is $\lambda^{*}>0$ with $\mu>1$ where the technological principle is expected to be introduced in a fraction $\Delta\left(\rho, d, \lambda^{*}\right)$ of all clusters and in a fraction $\Delta\left(\rho, d, \lambda^{*}\right) p\left(\rho, d, \lambda^{*}\right)$ of all final goods. It is the competition among innovators for profit flows that leads to this result. The historical record does show that the great innovation waves (the railroads, electricity, the microchip and internet) have all taken place at at time of plentiful finance and low real interest rates.

An increase in the technology-specific $(\rho)$ and and in the economy-specific diffusivity rate $(d)$ increases the investment in the discovery of the technology principle and in its diffusion within and between technology clusters thereby increasing the economy's growth rate. 


\section{A more general case}

We generalize the previous analysis in two ways. Firstly, we relax the assumption that all technologies are identical, and consider the case where some technologies are more easily propagated than others. Secondly, we consider the case where the number of innovators investing in the discovery and diffusion of technological principles is endogenously determined.

\subsection{Heterogeneous technological principles}

In the previous section any seed technology and its related technological principle appearing with probability $\phi\left(H^{*}\right)$ bear a time horizon measured by $\tau$ consequently defining a technology-specific discount factor. This assumption can be relaxed at no great cost by recognizing that innovations appear stochastically: they can be assumed to be drawn from a distribution reflecting the relative difficulty of each to be adaptable and transferable across heterogeneous goods. Simplifying somewhat the problem, the idea can be retained that some technologies are intrinsically more difficult to adopt by other sectors than others, while some are simpler, a stylized fact that we model by the length of the time span, $\tau$, it takes to bridge two technology clusters. Let there be an ordering of $1, \ldots, m, \ldots, M$ possible technologies with $\tau_{1}>\ldots>\tau_{m}>\ldots>\tau_{M}$ and the corresponding discount factors $\rho_{1}<\ldots<\rho_{m}<\ldots<\rho_{M}$.

The would-be innovator at the moment of investing in the discovery of the technological principle does not know the type of technology that will be discovered, but knows the probability distribution

$Q=\left\{q_{m}\right\}_{m=1}^{M}$ where $\operatorname{Pr}\left(\tau=\tau_{m}\right)=q_{m}$ and $\sum_{m=1}^{M} q_{m}=1$. We indicate with $Q^{\prime}>Q$ the probability distribution $Q^{\prime}$ that first order stochastically dominates $Q$.

As a consequence, for each $\lambda$ it is possible to find the critical technology that separates those technologies that have economy-wide impacts from those that have vanishing small ones. More formally, a critical technology $m^{*}$, if it exists, is defined as

$$
\begin{aligned}
& m \geq m^{*}(d, \lambda) \Longrightarrow \mu(d, \lambda)>1 \\
& m<m^{*}(d, \lambda) \Longrightarrow \mu(d, \lambda)<1
\end{aligned}
$$

where $m^{*}$ (and $\rho_{m}^{*}$ ) are increasing in $\lambda$ and decreasing in $d$. Thus, it is only for technologies above $m^{*}$ that diffusion across sectors is likely to be sustained leading to economy-wide effects. If this critical 
technology does not exist then we set $m^{*}=\infty$ and hence $\mu(d, \lambda)$ is always smaller than 1.

Once the technological principle has been discovered, the innovator solves problem (4) and thus for a given $\rho_{m}$ and $\lambda$ the value of the innovation of type $m$ can be determined $V\left(\rho_{m}, d, \lambda\right)$. In this case we also assume that $\theta$ is sufficiently large such that $V$ is always positive and $\rho_{m}$ and $d$ are sufficiently small such that $\psi\left(V, \rho_{m}, d, \lambda\right)$ is a contraction for each $m=1, \ldots, M$.

The innovator's problem to conjure up a seed innovation can now be set as:

$$
\Pi=\max _{H}\left\{\phi(H) \sum_{m=1}^{M} q_{m} V\left(\rho_{m}, d, \lambda\right)-H-F\right\}
$$

the FOC yielding $H^{*}(d, \lambda)$ and $\phi(d, \lambda)=\phi\left(H^{*}(d, \lambda)\right)$.

Taking the limit for $J \rightarrow \infty$

$$
\lim _{J \rightarrow \infty} \frac{N}{J} \sum_{s=1}^{G} \mu\left(\rho_{m}, d, \lambda\right)^{s}=\left\{\begin{array}{ccc}
\Delta\left(\rho_{m}, d, \lambda\right)>0 & \text { for } & m \geq m^{*}(d, \lambda) \\
0 & \text { for } & m<m^{*}(d, \lambda)
\end{array}\right.
$$

Let $\Gamma$ be the number of competing innovators, then in the limit for $J \rightarrow \infty$ the probability $\lambda$ of being ousted from the production of a single final good solves the following fixed point problem:

$$
\lambda=\left\{\begin{array}{cccc}
\Lambda(d, \Gamma, Q, \lambda) & \text { if } & m^{*}(d, \lambda) \leq M \\
0 & \text { if } & m^{*}(d, \lambda)>M
\end{array}\right.
$$

where $\Lambda(d, \Gamma, Q, \lambda) \equiv \Gamma \phi(d, \lambda) \sum_{m=m^{*}(d, \lambda)}^{M} q_{m} p\left(\rho_{m}, d, \lambda\right) \Delta\left(\rho_{m}, d, \lambda\right)$.

Proposition 2 If $r$ is sufficiently small then the solution to the fixed point problem (11) is $\lambda^{*}>0$ that solves

$$
\lambda^{*}=\Lambda\left(d, \Gamma, Q, \lambda^{*}\right)
$$

where $\lambda^{*}=\lambda(d, \Gamma, Q)$ is increasing in $d$ and $\Gamma$ and $\lambda\left(d, \Gamma, Q^{\prime}\right)>\lambda(d, \Gamma, Q)$ for $Q^{\prime}>Q$.

The economy's growth rate is

$$
g(d, \Gamma, Q)=\frac{\gamma-1}{\gamma} \Lambda\left(d, \Gamma, Q, \lambda^{*}\right)
$$

where technologies $m \geq m^{*}$ find an application in a fraction $\Delta\left(\rho_{m}, d, \lambda^{*}\right) p\left(\rho_{m}, d, \lambda^{*}\right)$ of firms, while technologies $m<m^{*}$ are introduced in a negligible fraction of firms. The growth rate $g$ is increasing in $d$ and $\Gamma$ and $g\left(d, \Gamma, Q^{\prime}\right)>g(d, \Gamma, Q)$ for $Q^{\prime}>Q$. 
Proof of Proposition 2. The Proof of Proposition 2 is similar to the one of Proposition 1. Consider a technology of type $m^{\prime}<M$. For a given value of $\rho_{m^{\prime}}$ there always exists a sufficiently small $r$ such that for $\lambda=0$ the incentive to invest in the diffusion of the innovation, and hence the amount invested, becomes infinitely large. Hence $\mu>1$ for each $m>m^{\prime}$ and hence $\lambda=0$ cannot be a solution to the fixed point problem (11).

Since $\Lambda\left(d, \Gamma, Q, \lambda^{*}\right)$ is increasing in $d$ and $\Gamma$ and $\Lambda\left(d, \Gamma, Q^{\prime}, \lambda^{*}\right)>\Lambda\left(d, \Gamma, Q, \lambda^{*}\right)$ for $Q^{\prime}>Q$ the result stated in the proposition follows.

The interpretation of these results is very similar to the ones in Proposition 1.

\subsection{Competing innovators and endogenous entry}

The number of competing innovators can then be made endogenous by introducing a free-entry condition. In this case, the probability of a seed innovation appearing is itself a function of the number of innovators just as is the expected value of an innovation. We assume that as long as the expected return from the investment in the discovery of the technology principle is positive, innovators enter the race to snatch a profit flow away from incumbent monopolists by paying the involved sunk cost $F$. Let us assume that $r$ is sufficiently small such that from Proposition 2 the arrival rate of a technological principle is $\phi\left(H^{*}(d, \Gamma)\right)$ and the value of the innovation of type $m$ is $V\left(d, \rho_{m}, \Gamma, Q\right)$ $=V\left(d, \rho_{m}, \lambda^{*}(d, \Gamma, Q)\right)$, then innovators enter as long as

$$
\sum_{m=1}^{M} q_{m} \phi\left(H^{*}(d, \Gamma)\right) V\left(d, \rho_{m}, \Gamma, Q\right)-H^{*}(d, \Gamma) \geq F
$$

For simplicity's sake we neglect the integer problem and assume that $\Gamma$ is real-valued. Hence, at the equilibrium condition (13) is satisfied with the equality sign. Resorting to the envelope theorem it can be concluded that since $V\left(d, \rho_{m}, \Gamma\right)$ is strictly decreasing in $\Gamma^{6}$, the left-hand side of this equation is strictly decreasing in $\Gamma$ insuring that a solution $\Gamma^{*}=\Gamma(d, Q)$ exists. A larger diffusivity $d$ and a $Q^{\prime}>Q$ increase the entrepreneur's expected profits flow but they also increase the probability of being ousted by a subsequent innovator; the overall effect of $d$ and $Q$ on entry is therefore ambiguous.

Given the distribution of the $M$ possible seed innovations and related technological principles, and defining $\mu\left(d, \rho_{m}, \Gamma^{*}(d)\right)=\mu\left(d, \rho_{m}\right)$, it is then possible to identify the technology $m^{*}$ such that $\mu\left(d, \rho_{m}\right)<1$ for each $m \geq m^{*}$ and $\mu\left(d, \rho_{m}\right)>1$ for each $m<m^{*}$. The following proposition

\footnotetext{
${ }^{6}$ From Proposition 2 we know that an increase in $\Gamma$ increases $\lambda^{*}$ and as a consequence decreases $V\left(d, \rho_{m}, \lambda^{*}\right)$
} 
holds. Let us define $\phi(d, \lambda(d, \Gamma(d, Q), Q))=\lambda(d, Q), p\left(\rho_{m}, d, \lambda(d, \Gamma(d, Q), Q)\right)=p\left(\rho_{m}, d, Q\right)$ and $\Delta\left(\rho_{m}, d, \lambda(d, \Gamma(d, Q), Q)\right)=\Delta\left(\rho_{m}, d, Q\right)$.

Proposition 3 For sufficiently low values of $r, \lambda^{*}$ is given by (12), $\Gamma^{*}$ is determined by the free entry condition (13) and the economy's growth rate is given by

$$
g(d, Q)=\frac{\gamma-1}{\gamma} \Gamma(d, Q) \phi(d, Q) \sum_{m=m^{*}}^{M} p\left(\rho_{m}, d, Q\right) \Delta\left(\rho_{m}, d, Q\right)
$$

If $d$ and $Q$ have a positive effect on entry then $g$ is increasing in $d$ and $g\left(d, Q^{\prime}\right)>g(d, Q)$ for $Q^{\prime}>Q$.

Proof of Proposition 3. If $d$ and $Q$ have a positive effect on entry then $\lambda^{*}=\lambda(d, \Gamma(d, Q), Q)=$ $\lambda(d, Q)$ is increasing in $d$ and $\lambda\left(d, Q^{\prime}\right)>\lambda(d, Q)$ for $Q^{\prime}>Q$. Hence, $\Lambda\left(d, \Gamma, Q, \lambda^{*}\right)$ in Proposition 2 is increasing in $d$ and $\Lambda\left(d, \Gamma, Q^{\prime}, \lambda^{*}\right)>\Lambda\left(d, \Gamma, Q, \lambda^{*}\right)$ for $Q^{\prime}>Q$ and the result stated in the Proposition follows.

Proposition 3 describes the growth rate of an economy where entrepreneurs invest in the discovery and the diffusion of innovations throughout the economy and the equilibrium number of entrepreneurs is determined endogenously through a free entry condition.

\section{Conclusions}

In this paper we have shown that technological convergence, that is, the process of diffusion of an original innovative principle across a heterogeneous economy, emerges as a result of the dynamic interplay between competition among entrepreneurs' for profit flows and entrepreneurial investment decisions. In particular, we have argued that diffusion rests on investment aimed at introducing and adapting a seed innovation into a cluster of firms sharing a degree of technological similarity. As a consequence of this initial effort a process of learning-to-adapt is likely to arise cumulating sufficient expertise to enable the original principle to cross-over to technologically distant clusters. Investment, therefore, lays the ground for success to breed more success. Diffusion is thus modeled as a branching process and the condition for its survival has been identified. It has, accordingly, been shown that below a precisely defined threshold in terms of the expected number of clusters that at each step are likely to be reached the process dies out while above, it stands a positive probability of diffusing 
over a significant part of the whole economy. Investment to entice diffusion through learning-toadapt as well as to conjure up the original seed innovation is determined as a result of innovators attempting to maximize their innovation value. The framework within which diffusion unfolds is shaped as a monopolist-incumbent, innovation-searching entrant confrontation. This generates an equilibrium between the incentive to invest and the probability of an innovation being introduced. This probability has, in turn, been endogenously determined as a function of the probability of diffusion to penetrate the whole economy. We have shown that the entrepreneur's investment in the diffusion of the technological principle together with the competition among entrepreneurs for profits flows sets the economy in a state where technological convergence occurs and general purpose technologies emerge with a positive probability characterizing the economy's growth rate.

\section{Appendix}

\section{Generalization of Assumption 1.}

Lemma 2 Assume that the number $k$ of clusters that are reachable be a random variable, the cumulative distribution function of which, $F(k, n, d)$, depends on the the number $n$ of adoptions within the cluster, on the diffusivity parameter d and is convex and decreasing with respect to both variables. It, then, follows that mean number $\mu(I, d)$ is a concave increasing function with respect to $I$ and $d$.

Assume that $F(K, n, d)=1$.

Proof. The mean value of the number $k$ of reachable new clusters, conditional on the number $n$ of innovations, is

$$
E(k \mid n)=\int_{0}^{K} k d_{k} F(k, n, d)
$$

which is an increasing concave function of $n$ and $d$.

On the other hand, by lemma 2 the probability mass function of the random variable $n$ is

$$
\operatorname{pr}(n=j)=\left(\begin{array}{c}
N \\
j
\end{array}\right) p(I)^{j}(1-p(I))^{N-j}
$$


Hence $\mu=E(k)$ is

$$
\mu(I, d)=\sum_{j=0}^{N}\left(\begin{array}{c}
N \\
j
\end{array}\right) p(I)^{j}(1-p(I))^{N-j} E(k \mid j)
$$

deriving we have

$$
\begin{aligned}
& \frac{\partial \mu(I, d)}{\partial I}=\sum_{j=0}^{N}\left(\begin{array}{c}
N \\
j
\end{array}\right) k p(I)^{j-1}(1-p(I))^{N-j} E(k \mid j) \frac{\partial p}{\partial I} \\
& -\sum_{j=0}^{N}\left(\begin{array}{c}
N \\
j
\end{array}\right)(N-j) p(I)^{j}(1-p(I))^{N-j-1} E(k \mid j) \frac{\partial p}{\partial I}
\end{aligned}
$$

rearranging terms

$$
\begin{gathered}
\frac{\partial \mu(I, d)}{\partial I}=\sum_{j=0}^{N-1}\left(\begin{array}{c}
N \\
j
\end{array}\right) p(I)^{j}(1-p(I))^{N-j-1} \\
(N-j)(E(k \mid j+1)-E(k \mid j)) \frac{\partial p}{\partial I}
\end{gathered}
$$

By the properties of function (14) the right hand side of (15) is positive: hence $\mu(I, d)$ is increasing in $I$.

The second derivative can be analogously calculated

$$
\begin{gathered}
\frac{\partial^{2} \mu(I, d)}{\partial I^{2}}= \\
\sum_{j=0}^{N-2}\left(\begin{array}{c}
N \\
j
\end{array}\right) p(I)^{j}(1-p(I))^{N-j-2}(N-j)(N-j-1) \\
(E(k \mid j+2)-2 E(k \mid j+1)+E(k \mid j))\left(\frac{\partial p}{\partial I}\right)^{2} \\
+\sum_{j=0}^{N-1}\left(\begin{array}{c}
N \\
j
\end{array}\right) p(I)^{j}(1-p(I))^{N-j-1} \\
(N-j)(E(k \mid j+1)-E(k \mid j)) \frac{\partial^{2} p}{\partial I^{2}}
\end{gathered}
$$

Again by the the properties of function (14), the right hand side of (16) is negative: hence $\mu$ is concave in $I$.

The proof of the properties of $\mu(I, d)$ as a function of $d$ can be proved along the same lines. 


\section{References}

[1] Andergassen R., F. Nardini and M. Ricottilli, 2006. Innovation Waves, Self-organised Criticality and Technological Convergence. Journal of Economic Behavior and Organization 61, 710 - 728.

[2] Andergassen R., F. Nardini and M. Ricottilli, 2009. Innovation and growth through local and global interaction. Journal of Economic Dynamics and Control 33, 1779 - 1795.

[3] Arenas, A., A. Díaz-Guilera, C.J. Pérez and F. Vega-Redondo, 2002. Self-organized criticality in evolutionary systems with local interaction. Journal of Economic Dynamics and Control 26, $2115-2142$.

[4] David, P. A, 1991. "Computer and Dynamo: The Modern Productivity Paradox in a Not-TooDistant Mirror." In Technology and Productivity: The Challenge for Economic Policy, 315-47. Paris: OECD.

[5] Fai, F. and N. von Tunzelmann, 2001. Industry-specific competencies and converging technological systems: evidence from patents. Structural Change and Economic Dynamics 12, 141-170.

[6] Goldfarb, B., 2005. Diffusion of general-purpose technologies: understanding patterns in the electrification of US Manufacturing 1880-1930. Industrial and Corporate Change, 14, 5, 745-773.

[6] Helpman, E., and M. Trajtenberg, 1998. "A Time to Sow and a Time to Reap: Growth Based on General Purpose Technologies." In General Purpose Technologies and Economic Growth, edited by E. Helpman, 55-83. Cambridge, MA: MIT Press.

[7] Helpman, E. Ed., 1998. General Purpose Technologies and Economic Growth. Cambridge, MA: MIT Press.

[8] Rosenberg, N., 1976. "Technological Change in the Machine Tool Industry: 1840-1910." In Perspectives on Technology, 9-31. Cambridge: Cambridge University Press.

[9] Rosenberg, N., 1982. "Technological Interdependence in the American Economy." In Inside the Black Box: Technology and Economics, 55-80. Cambridge: Cambridge University Press.

[10] Rosenberg, N. and M. Trajtenberg, 2004. A General-Purpose Technology at Work: The Corliss Steam Engine in the Late- Nineteenth-Century United States. Journal of Economic History 64, 1 -39 . 
[11] Silverberg G. and B. Verspagen, 2005. A percolation model of innovation in complex technology spaces. J Econ Dyn Control 29:225-244 


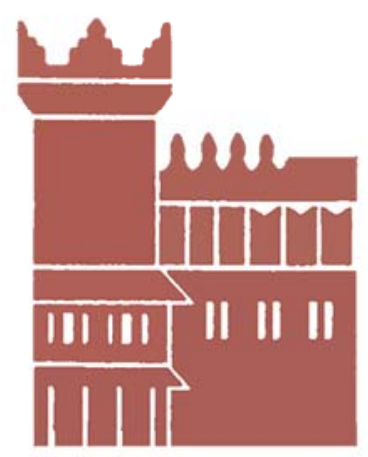

Alma Mater Studiorum - Università di Bologna DEPARTMENT OF ECONOMICS

Strada Maggiore 45

40125 Bologna - Italy

Tel. +39051 2092604

Fax +390512092664

http://www.dse.unibo.it 